\title{
Numerical and Experimental Analysis of Plasma Flow Control Over a Hump Model
}

\author{
Chuan $\mathrm{He}^{*}$ and Thomas C. Corke ${ }^{\dagger}$ \\ University of Notre Dame, Notre Dame, IN 46556 \\ Mehul P. Patel ${ }^{\ddagger}$ \\ Orbital Research Inc., Cleveland, OH 44103
}

\begin{abstract}
Turbulent flow separation over a wall-mounted hump model and its control using plasma actuators were studied numerically and experimentally. The hump model chosen in this study was that used in a 2004 NASA Langley CFD validation workshop for turbulent separation control using synthetic jets. The numerical simulations solved the Reynoldsaveraged Navier-Stokes equations using Fluent. Different turbulence models based on $k-\varepsilon$, $k-\omega$ and $S A$ were investigated. All turbulence models did a reasonable good job predicting the overall features of the flow fields, but only the $k-\varepsilon$ model agreed well with the experimental data in terms of the skin friction coefficient. A body force model was used to simulate the effect of the plasma actuator. These results showed that the plasma actuator could be effective in controlling the turbulent flow separation over the hump. Both spanwise and streamwise plasma actuator configurations were investigated experimentally at a $R e_{c}=288 \mathrm{~K}$. A smoke wire was used to visualize counter-rotating vortices generated by the streamwise plasma actuators. An optimum spacing between the exposed electrodes of the streamwise plasma actuator was found. Surface pressure coefficients demonstrate both streamwise and spanwise configurations work well for turbulent separation control.
\end{abstract}

\section{Introduction}

Currently a great deal of interest within the community is to utilize the emerging flow control technology to design revolutionary Uninhabited Air Vehicles (UAVs) without moving control surfaces while still maintaining controlled flight. Corke et al. ${ }^{1}$ proposed a plasma flow control optimized airfoil concept. It uses a laminar airfoil design that maintains a favorable pressure gradient over as much of the upper surface as possible and incorporates a separation ramp at the trailing edge that can be manipulated by a plasma actuator in order to control lift. At lower angles of attack such as during cruise flight, a laminar boundary layer is maintained until it reaches a separation ramp. The separation in this case is a laminar which can easily be controlled with an unsteady plasma actuator at very low power levels. At higher angles of attack such as take-off and landing, transition to turbulence may occur somewhere before the ramp due to adverse pressure gradients, so the flow approaching the ramp could be turbulent. Thus plasma actuators for turbulent separation control are needed for all the possible flight regimes.

Plasma actuators have been under development for some time at the University of Notre Dame in the Center for Flow Physics and Control. ${ }^{2}$ Most of the applications have focused on laminar separation control, such as leading-edge separation control on wing sections ("plasma slats"), ${ }^{3}$ control of dynamic stall on oscillating airfoils related to helicopter rotors, ${ }^{4,5}$ and separation control for low-pressure turbine blades. ${ }^{6,7}$ For these, optimum frequency periodic disturbances generated by the plasma actuator successfully suppressed or delayed the flow separation. Instability mechanisms sometimes plays a role in this process, but it is not clear how well these approaches work when the approaching flow is turbulent. The object of the work

* Ph.D. Candidate.

$\dagger$ Clark Chair Professor, Associate Fellow AIAA.

$\ddagger$ Senior Member, AIAA. 


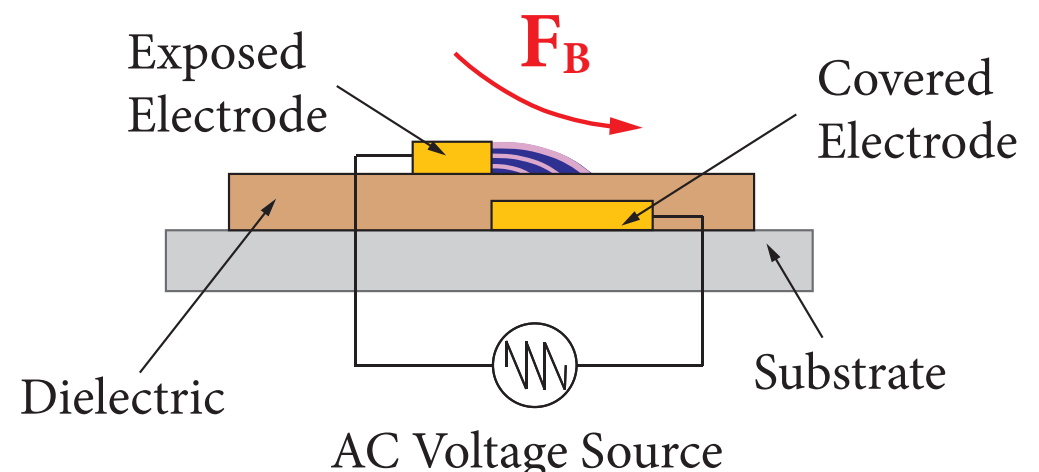

Figure 1. Schematic drawing of asymmetric electrode arrangement for plasma actuators.

presented here is to understand turbulent separation and explore the capability of plasma actuators in its control.

The single-dielectric barrier discharge (SDBD) plasma actuator consists of thin electrodes separated by a dielectric insulator. One of the electrodes is typically exposed to the air. The other electrode is fully covered by a dielectric material. A schematic illustration is shown in Figure 1. A high voltage a.c. potential is supplied to the electrodes. When the a.c. amplitude is large enough, the air ionizes in the region of the largest electric potential. This generally begins at the edge of the electrode that is exposed to the air, and spreads out over the area projected by the covered electrode. The ionized air (plasma) in the presence of the electric field produced by the electrodes results in a body force on the ambient air. Details of the physics and mechanism of the plasma actuator are provided by Enloe et al. 8,9

The body force vector can be tailored through the design of the electrode arrangement. Turbulent separation control can be achieved either by the injection of high momentum fluid, or by circulating high momentum fluid in the free-stream or outer part of the boundary layer towards the wall. The spanwise electrode arrangement which is often used in laminar separation control, provides the former approach. The body force generated by this arrangement accelerates fluid towards the separation location to overcome the adverse pressure gradient. To enhance boundary layer mixing, a streamwise electrode arrangement was designed in order to generate a pair of counter-rotating vortices that are intended to circulate high momentum fluid from outer part of the boundary layer towards the wall to delay or suppress flow separation.

A wall-mounted hump model was selected in this study as a canonical turbulent separated flow field. It consists of a relatively long fore-body and a short separation concave ramp near the aft part of the model. This shape was chosen because the separation location is not sensitive to Reynolds number and detailed experimental data are well-documented in the literature. ${ }^{10}$ This shape was used in a 2004 NASA Langley CFD validation workshop for synthetic jets and turbulent separation control. ${ }^{11}$ A broad range of numerical techniques such as RANS, LES and DNS have been performed to predict the separated flow field and its control. ${ }^{12}$ With all of this published information, it made an excellent test case to evaluate our simulations and make a comparison of the effectiveness of the plasma actuator.

In this study, the Reynolds-averaged Navier-Stokes equations were solved to predict the turbulent flow separation over the hump model. Detailed comparisons of surface pressure coefficient, skin friction coefficient, mean velocity profiles and Reynolds stress profiles are made against the different turbulence models and previous published results. ${ }^{11}$ The plasma actuator effect is introduced into the simulations through a body force model. ${ }^{13}$ The numerical simulations with and without the plasma actuator are then compared to the results from wind tunnel experiment on the hump model.

\section{Computational Methodology}

A segregated solver was used to solve two dimensional Reynolds-averaged Navier-Stokes equations coupling a model for the plasma actuator effect. It is one of the solution algorithms provided by the Fluent CFD modeling package. Using this approach, the $u$ and $v$ momentum equations are each solved sequentially in order to update the velocity field. Since the obtained velocities may not satisfy the continuity equation 
Velocity Inlet BCs at $\mathrm{x} / \mathrm{c}=-6.39$

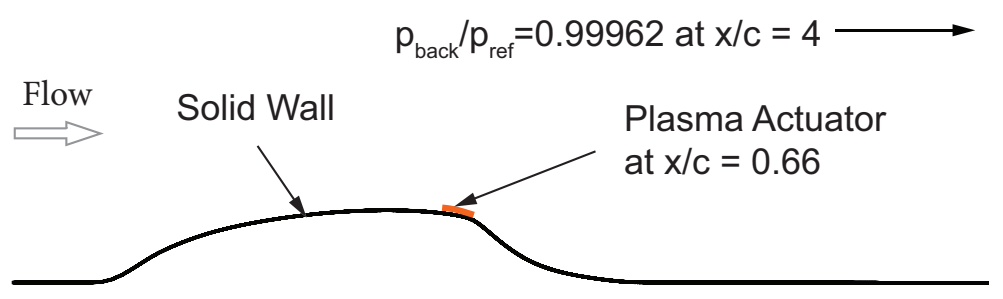

(a)



(b)

Figure 2. (a) Boundary conditions for numerical simulation and location of plasma actuator.

(b) Computational grids.

locally, a "Poisson-type" equation for the pressure correction is derived from the continuity equation and the linearized momentum equations. This pressure correction equation is then solved to obtain the necessary corrections to the pressure and velocity fields and the face mass fluxes such that continuity is satisfied. In the segregated solution method, each discrete governing equation is linearized implicitly with respect to that equation's dependent variable. This results in a system of linear equations with one equation for each cell in the domain. A point implicit (Gauss-Seidel) linear equation solver is used in conjunction with an algebraic multi-grid (AMG) method to solve the system equations for the dependent variable in each cell. Fluent uses a control-volume-based technique to discretize the governing equations to algebraic equations that can be solved numerically. A second order upwind scheme was chosen for the convection terms and the viscous terms. The SIMPLE algorithm was used for pressure-velocity coupling. ${ }^{14}$

The flow configuration and boundary conditions are shown in Figure 2 (a). This configuration is one of the test cases considered in the NASA Langley workshop on CFD validation of synthetic jets and turbulent separation control. The hump is the upper surface of a modified Glauert airfoil which consists of a relatively long fore body and a short separation ramp at the trailing edge. The hump has a physical chord length of $c=420 \mathrm{~mm}$ and a maximum height of $53.7 \mathrm{~mm}$. The inlet is located at $x / c=-6.39$ where the free-stream Mach number is set to be $0.1\left(U_{r e f}=34.6 \mathrm{~m} / \mathrm{s}\right)$. The outlet is located at $x / c=4.0$ where the pressure is set at $p / p_{\text {ref }}=0.99962$. The top-wall is located at $x / c=0.9$ with a slight modification which accounts for the blockage due to the presence of the end plates. Solid wall conditions are applied at top-wall, floor and hump surface. The plasma actuator is placed at $x / c=0.66$, near where the flow is predicted to separate.

The computational grid was generated using Gambit, Fluent's geometry and mesh generation software. The grid is a two dimensional structured grid as shown in Figure $2(\mathrm{~b})$. It has $412 \times 46(\mathrm{x} \times \mathrm{y})$ grid points. 
Grid dependence was carried out in earlier study, solution no longer changed with further grid refinement. Based on this study, the mesh is believed to have sufficient resolution to capture the features of flow fields over the hump.

In order to simulate the plasma actuator effect, a body force model was created. ${ }^{13}$ The body force per volume of plasma is a vector, given as

$$
\vec{F}_{B}=\left(-\frac{\epsilon_{O}}{\lambda_{D}} \phi\right) \vec{E}
$$

where $\epsilon_{o}$ is the permittivity of free space $\left(8.854 \times 10^{-12} \mathrm{~F} / \mathrm{m}\right), \lambda_{D}$ is the Debye length, $\phi$ is the electric potential, and $\vec{E}$ is the electric field vector, where

$$
\vec{E}=-\vec{\nabla} \phi
$$

The Debye length is the characteristic length for electrostatic shielding in a plasma. It varies with plasma density and temperature as

$$
\frac{1}{\lambda_{D}^{2}}=\frac{e^{2} n_{o}}{\epsilon_{o}}\left(\frac{1}{k T_{i}}+\frac{1}{k T_{e}}\right)
$$

where $k T_{i}$ and $k T_{e}$ are the ion and electron temperature, $n_{o}$ is the plasma density, and e is the elementary charge.

The electrostatic potential $\phi$ is given in the following equation, which is derived from one of Maxwell equations.

$$
\nabla(\epsilon \nabla \phi)=s(x, y) \frac{1}{\lambda_{D}} \phi
$$

where $s(x, y)$ is a rectangular sub-domain in the air over the plasma actuator that is 1 at the intersection of the exposed and covered electrode and decrease exponentially to 0 at the downstream edge of the covered electrode and 0 over the exposed electrode.

The solution of the spatial distribution for the voltage potential is substituted into Eq 1 and Eq 2 to obtain the spatial body force, $\vec{F}_{B}$. This added body force is applied to the computational grid points at the location of the actuator in the computational domain. For the steady actuator effect, the body force is constant at very iteration. For the unsteady actuator effect, the unsteady period during which the actuator is on and off is discretized into the time steps.

\section{Numerical Results}

The surface pressure coefficients obtained from the simulations compared to the experimental results of Greenblatt et al. ${ }^{10}$ are shown in Figure 3. Basically, the surface pressure coefficient reflects the overall features of the flow field. The flow decelerates when approaching the hump's leading-edge. Immediately downstream of the leading-edge, the flow accelerates up to mid-chord region due to a favorable pressure gradient. Afterwards the sharp drop in the $-C_{p}$ over the hump near $x / c=0.66$ indicates a flow separation. The flow remains separated over the concave ramp and re-attaches downstream of the trailing-edge. $k-\varepsilon$ model , $S S T k-\omega$ model and $S A$ model were used in the simulations for the uncontrolled case. In the region of $0.4<x / c<0.66$, only $S S T k$ - $\omega$ model agree with the experimental results very well. The other two models predicted a slightly lower pressure. All the turbulence models slightly miss the pressure level in the separation region. Otherwise the general features are consistent with the experimental results.

Skin friction coefficients are shown in Figure 4. The horizontal dash line represents $C_{f}=0$, which provides the border between the separated and re-attached flow. The $k-\varepsilon$ model shows a favorable agreement with experimental data over the whole hump, thus the location of re-attachment and bubble size obtained from $k$ - $\varepsilon$ model are more accurate than the others. All three turbulence models predicted the separation point accurately.

Mean velocity profiles were extracted from the simulation results at three typical locations: $x / c=0.8$, $x / c=1.0$ and $x / c=1.2 . x / c=0.8$ and $x / c=1.0$ locate inside the separation bubble and $x / c=1.2$ locates downstream of the re-attachment location. Mean velocity profiles at these representative locations are compared with experimental data for the uncontrolled case in Figure 5 . Velocity profiles at $x / c=0.8$ and $x / c=1.0$ were predicted reasonably well, but $S S T k-\omega$ model and $S A$ model compared poorly with experimental data at $x / c=1.2$. One of the possible reasons is that both models predict a longer separation bubble, thus the distance between the re-attachment and measurement location becomes shorter and the 


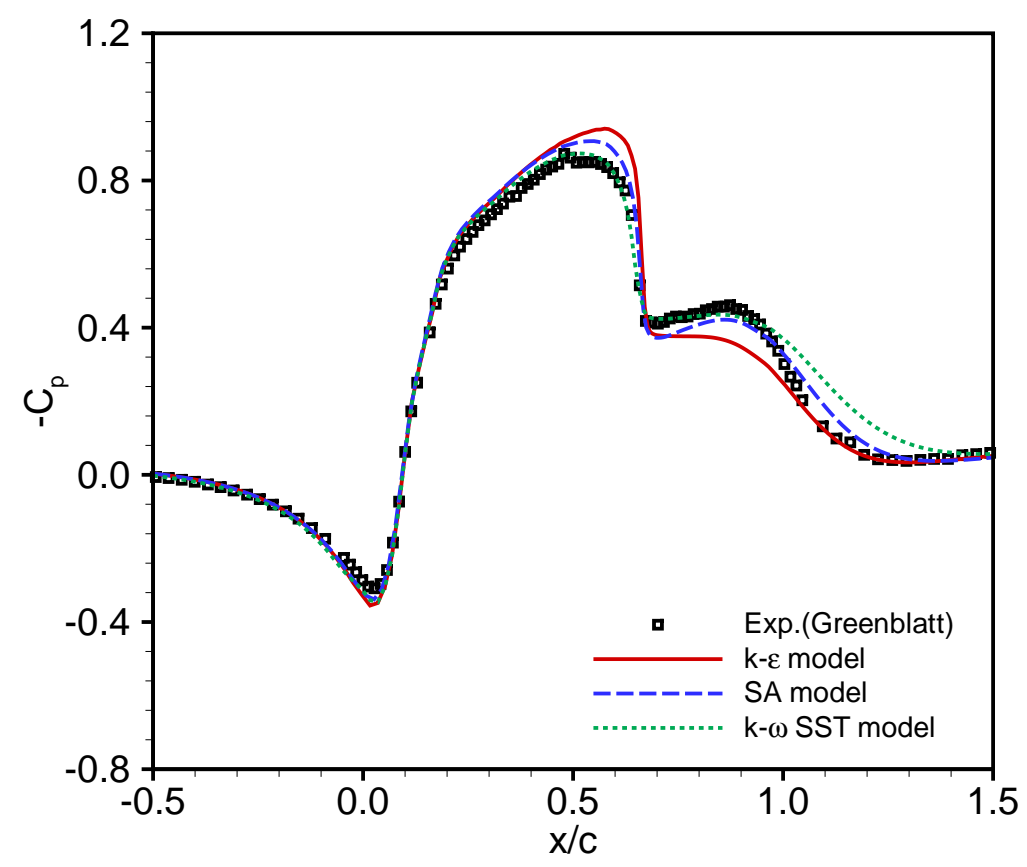

Figure 3. Surface pressure coefficients predicted by three turbulence models compared to experimental data for base flow.

boundary layer has not fully recovered at $x / c=1.2 . \quad k-\varepsilon$ predicted a little lower velocity in the region $0.05<\left(y-y_{\text {wall }}\right)<0.10$ at all locations, otherwise the agreement with experimental data is fairly good.

Representative Reynolds stress profiles for the uncontrolled case are shown in Figure 6. All the turbulence models predicted the Reynolds stress to be too small at all locations. In the region $0.05<\left(y-y_{\text {wall }}\right)<0.10$, the $k$ - $\varepsilon$ model predicted lower Reynolds stress than the other models, which might be one of the reasons that $k-\varepsilon$ model predicted lower velocity levels than the $k-\omega$ and $S A$ models in the same region.

In order to simulate the plasma actuator effect, Eq. 4 was separately solved numerically, then the solution of electric potential was substituted into Eq. 1 and Eq. 2 to calculate the body force. Figure 7 shows the body force magnitude as constant level contours. Both steady actuation and unsteady actuation were simulated in this study. For the unsteady actuator, the forcing frequency was set so that $F^{+}=f L_{s e p} / U_{\infty}=1$, where $L_{\text {sep }}$ is the streamwise extent of the flow separation. Our past experiments ${ }^{3}$ have indicated that the unsteady frequency at $F^{+}=1$ is optimum for separation control.

Streamlines colored by streamwise velocity in Figure 8 qualitatively show the effectiveness of plasma actuators in steady and unsteady operation. The red color and blue color represent high and low velocity respectively. Clearly the steady and unsteady actuation reduced the bubble size significantly. This is seen as a shortening of the distance to the re-attachment location, as well as the upstream movement of the center of circulation.

A comparison of surface pressure coefficients for the base flow and actuator cases is shown in Figure 9. When steady or unsteady actuation was on, the body force produced by plasma actuator locally accelerated the ambient air, which caused a local pressure drop. The sharp decline in the $C_{p}$ level at $x / c=0.66$ is indicative of this effect.

Both steady and unsteady actuation improved the pressure recovery in the separation region. The steady actuation was slightly better than the unsteady actuation. This is quite different from the results in laminar separation control where unsteady actuator operation at $F^{+}=1$ has always been found to be better than steady actuator operation. Examples for leading edge separation control by Post and Corke ${ }^{2,4}$ and Corke et al. ${ }^{3}$ support this observation.

A comparison of skin friction coefficients for the base flow and the actuator cases in Figure 10 demonstrate 
the effectiveness of plasma actuator more clearly. Again the horizontal dash line represents $C_{f}=0$, which defines the border between the separated and attached flow. The separation locations did not change for the steady and unsteady actuation while the re-attachment locations moved from $x / c=1.1$ to $x / c=1.02$ and $x / c=1.04$ respectively. Consequently the separation bubble reduced in length with the actuator on as previously indicated in Figure 8.

\section{Experimental Setup}

\section{A. Wind Tunnel and Hump Model}

The experiments were conducted in one of the subsonic wind tunnels in the University of Notre Dame Center for Flow Physics and Control (FlowPAC) in the Hessert Laboratory . The facility is an open-return blowdown wind tunnel with a $40.64 \mathrm{~cm}$ (16 in)wide by $40.64 \mathrm{~cm}$ (16 in) high by $182.88 \mathrm{~cm}$ (6 ft) long test section. The tunnel consists of a removable inlet having a series of 12 screens followed by a $24: 1$ contraction that attaches to the test section. The test section is equipped with a clear Plexiglas side-wall that allows optical access to view the model for flow visualization. The back wall of the test section has a removable panel to allow access into the test section. A schematic illustration of the subsonic wind tunnel and test section is shown in Figure 11.

A $38.1 \mathrm{~cm}$ wide by $162.56 \mathrm{~cm}$ long flat Plexiglas plate was mounted $10.16 \mathrm{~cm}$ above the floor of the test section, so the test section height became $30.48 \mathrm{~cm}$. The hump model was mounted on the top of the flat plate. The flat plate extended $82 \mathrm{~cm}$ upstream of the leading-edge and $42 \mathrm{~cm}$ downstream of the trailing-edge of the hump model. A sand paper strip was attached to the elliptical leading-edge of flat plate in order to trip the flow and achieve a fully-developed turbulent boundary layer downstream. Two end-mounted plates were designed to produce two dimensional flow field over the hump. Each end plate was $82 \mathrm{~cm}$ long and 23.5 $\mathrm{cm}$ high.

A photograph of the hump model is show in Figure 12. It has a $42 \mathrm{~cm}$ chord and a $35.6 \mathrm{~cm}$ span. Its maximum thickness is $5.37 \mathrm{~cm}$. The model was precisely machined from an aluminum plate using a numerical-controlled machine. It was equipped with 48 center-span static pressure ports. A scanning valve was used to direct the static pressure port outputs to a differential pressure transducer.

\section{Experimental Results}

Both spanwise and streamwise plasma actuator configurations were investigated in this study. The spanwise plasma actuator configuration is the one shown in Figure 12. The copper electrodes were separated by three layers of 5-mil (dark colored) Kapton film. The edges of the electrodes were aligned in the spanwise direction. The electrodes were made from $0.0254 \mathrm{~mm}$ thick copper foil tape. They were overlapped by a small amount (of the order of $1 \mathrm{~mm}$ ), in order to ensure a uniform plasma in the full spanwise direction. The junction of the exposed electrode and the covered electrode was placed precisely on the separation location $(x / c=66 \%)$.

The experiments were conducted at a $10 \mathrm{~m} / \mathrm{s}$ free-stream speed. This gave a chord Reynolds number of $288 \mathrm{~K}$. The actuator ran in steady operation. The frequency of the a.c. voltage supplied to the electrodes was approximately $1.3 \mathrm{kHz}$. The precise frequency was tuned to minimize the overall power in the actuator electronics. The a.c. voltage amplitude to the electrodes was $36 \mathrm{kV} V_{p-p}$.

The results are presented in Figure 13. The red square symbols represent the base flow, and the blue circle symbols represent the steady plasma actuator case. The spanwise plasma actuator was found to improve the separation recovery significantly.

The streamwise plasma actuator configuration was also investigated. The schematic illustration and photograph of the streamwise plasma actuators are shown in Figure 14 (a) and (b). The covered electrode ran across most of the span of the hump model. The width (b) of the covered electrode was $0.5 \mathrm{in}$. It was positioned at $1.75 \mathrm{in}$ upstream of separation location $(x=1.75 \mathrm{in})$. Several spacings $(\mathrm{z})$ between the exposed electrodes were studied. These ranged from $0.5 \mathrm{in}$ to $3.0 \mathrm{in}$. A $0.25 \mathrm{in}$ thick white-colored Teflon sheet (seen in Figure 14) was used to separate the exposed electrodes and the covered electrode.

The streamwise plasma actuator was expected to generate streamwise-oriented counter-rotating vortices similar to those produced by passive delta-wing vortex generators. Flow visualization was used to verify this idea. 
The flow visualization photograph in Figure 15 (a) was taken when the laser sheet and smoke sheet were in the same plane. There were two large vortices generated by the outer edges of plasma actuators. In addition, two vortices were generated by the inner edges. Because the inner edges were close, the inner vortices interacted with each other, and rolled up and formed two smaller vortices which are not as clearly seen in the photograph.

The photograph in Figure 15 (b) was taken when the laser sheet was raised above the smoke sheet. With this arrangement, three long smoke streaks were captured. These streaks are due to the rotating vortices which brought the smoke from the lower level up to the higher level of the laser light sheet.

The spacing between the exposed electrodes was found to control the size of the generated streamwise vortices. We next determined if an optimum spacing existed for turbulent separation control.

The effect of the spacing of the streamwise-oriented electrodes of the plasma actuator was investigated experimentally on the hump model. The result is shown in Figure 16. For this the spacing was varied from 0.5 to 3.0 inches. The free-stream speed was the same as the other experiments, namely $10 \mathrm{~m} / \mathrm{s}$. In these cases the frequency of the a.c. voltage supplied to the electrodes was approximately $2.3 \mathrm{kHz}$, with the precise frequency again tuned to minimize the overall power. The a.c. voltage amplitude to the electrodes was $48 k V_{p-p}$.

Figure 16 shows the change in the integrated static pressure in the separated region over the hump model as a function of the spacing between the streamwise-oriented electrodes. The results indicate a clear optimum at a spacing of approximately 1.5 inches. It is expected that the optimum spacing scales with the approaching boundary layer thickness. Future investigations will verify this.

The surface pressure coefficient distribution over the concave portion of the hump model for the optimum 1.5 in spacing is presented in Figure 17. This is labeled as $\operatorname{STPA}(1.5 \mathrm{in}$.$) in the figure. We observe that the$ streamwise-oriented plasma actuators improved the pressure recovery in the separation region, moving the re-attachment location upstream and reducing the size of the separation bubble. Also shown for reference in Figure 17 is the pressure coefficient distribution for the spanwise oriented plasma actuator. This is labeled SPPAs. This was found to produce a better pressure recovery than the streamwise-oriented actuator and therefore is still preferred for controlling turbulent flow separation.

\section{Conclusions}

Numerical simulations using a Reynolds-averaged Navier-Stokes solver were used to predict the flow field over a wall-mounted hump model. A $k-\varepsilon$ turbulence model predicted surface pressure and skin friction coefficient distributions, velocity profiles and Reynolds stress profiles in reasonably good agreement with experimental data. The plasma actuator effect was simulated through a body force model. Results indicate that plasma actuators are quite capable of turbulent flow separation control.

Experiments were conducted to confirm the simulation results. Both spanwise and streamwise plasma actuator configurations were investigated at a chord Reynolds number of $R e_{c}=288 \mathrm{~K}$. The surface pressure coefficients demonstrate that both configurations increase the pressure level in the separation region, and significantly reduced the size of the separation bubble. For the same conditions, the steady spanwise-oriented plasma actuator performed the best.

The spanwise plasma actuator can be used in either laminar separation control (unsteady operation) or turbulent separation control (steady operation) depending on the state of the flow field. Such a change in operation is fully electronic without any physical modification of the actuator.

For the streamwise plasma actuator, it can be placed upstream of the separation location unlike the spanwise plasma actuator. Therefore it may be more useful for separated flows in which the separation location changes with flight conditions.

The final goal of this work is to incorporate a separation ramp near the trailing-edge of an airfoil so that it can be manipulated by plasma actuators for flight control. The bench marking of the simulation tools for the flow field and plasma actuator effect that was presented here is a necessary step in the design of optimum airfoil shapes that exploit this application.

\section{Acknowledgement}

The authors are pleased to acknowledge support from Orbital Research Inc. under an SBIR Phase II Contract. 


\section{References}

${ }^{1}$ Corke, T., Mertz, B., and Patel, M., "Plasma Flow Control Optimized Airfoil," AIAA Paper 2006-1208.

${ }^{2}$ Corke, T. and Post, M., "Overview of Plasma Flow Control: Concepts, Optimization, and Application," AIAA Paper 2005-0563.

${ }^{3}$ Corke, T., He, C., and Patel, M., "Plasma flaps and slats: an application of weakly-ionized plasmas as wing flow control devices," AIAA Paper 2004-2127.

${ }^{4}$ Post, M. and Corke, T., "Separation control on high angle of attack airfoil using plasma actuators," AIAA J., Vol. 42, No. 11, 2004, pp. 2177, also AIAA Paper 2003-1024.

${ }^{5}$ Post, M. and Corke, T., "Separation control using plasma actuators - atationary and oscillating airfoils," AIAA Paper 2004-0841.

${ }^{6}$ Huang, J., Corke, T., and Thomas, F., "Plasma actuators for separation control of low pressure turbine blades," AIAA J., Vol. 44, No. 1, 2006, pp. 51.

${ }^{7}$ Huang, J., Corke, T., and Thomas, F., "Unsteady Plasma Actuators for Separation Control of Low Pressure Turbine Blades," AIAA J., Vol. 44, No. 7, 2006, pp. 1477.

${ }^{8}$ Enloe, L., McLaughlin, T., VanDyken, R., Kachner, K., Jumper, E., and Corke, T., "Mechanisisms and Response of a Single Dielectric Barrier Plasma Actuator: Plasma Morphology," AIAA J., Vol. 42, No. 3, 2004, pp. 589.

${ }^{9}$ Enloe, L., McLaughlin, T., VanDyken, R., Kachner, K., Jumper, E., Corke, T., Post, M., and Haddad, O., "Mechanisisms and Response of a Single Dielectric Barrier Plasma Actuator: Geometric Effects," AIAA J., Vol. 42, No. 3, 2004, pp. 585.

${ }^{10}$ Greenblatt, D., Paschal, K., Schaeffler, N., Washburn, A., Harris, J., and Yao, C., "A separation control CFD validation test case. Part 1: baseline and steady suction," AIAA Paper 2004-2220.

${ }^{11}$ Rumsey, C., Gatski, T., Sellers, W., Vatsa, V., and Viken, S., "Summary of the 2004 CFD Validation Workshop on Synthetic Jets and Turbulent Separation Control," AIAA Paper 2004-2217.

${ }^{12}$ You, D., Wang, M., and Moin, P., "Large-Eddy Simulation of Flow over a Wall-Mounted Hump with Separation Control," AIAA J., Vol. 44, No. 11, 2006, pp. 2571.

${ }^{13}$ Orlov, D. and Corke, T., "Numerical Simulation of Aerodynamic Plasma Actuator Effects," AIAA Paper $2005-1083$.

${ }^{14}$ Fluent 6.2 User's Guide, Fluent Inc., 2005.

${ }^{15}$ Corke, T., Jumper, E., Post, M., Orlov, D., and McLaughlin, T., "Application of Weakly-Ionized Plasma as Wing Flow-Control Devices," AIAA Paper 2002-0350. 


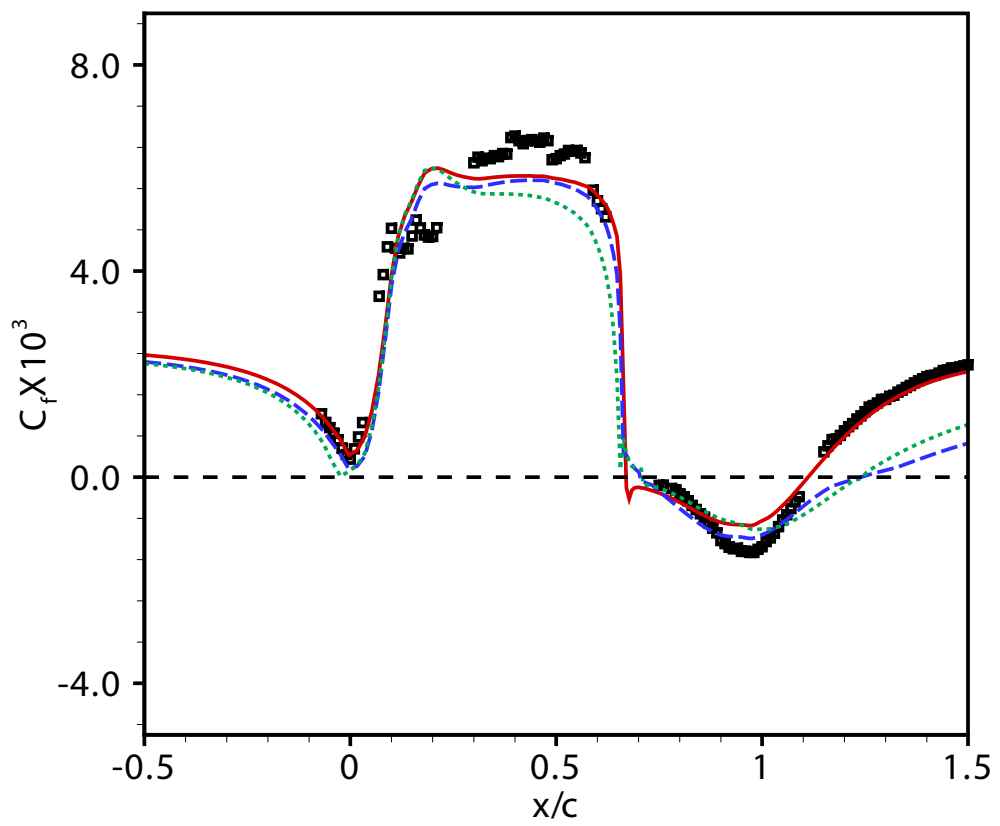

Figure 4. Skin friction coefficients predicted by three turbulence models compared to experimental data for base flow.

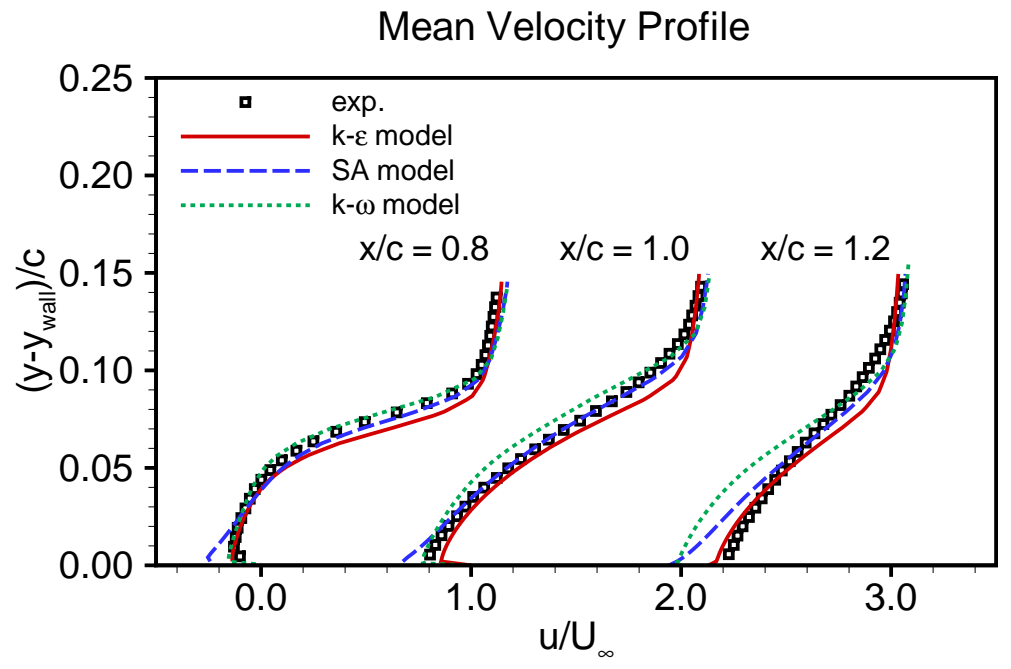

Figure 5. Mean streamwise velocity profile predicted by three turbulence models for base flow. 


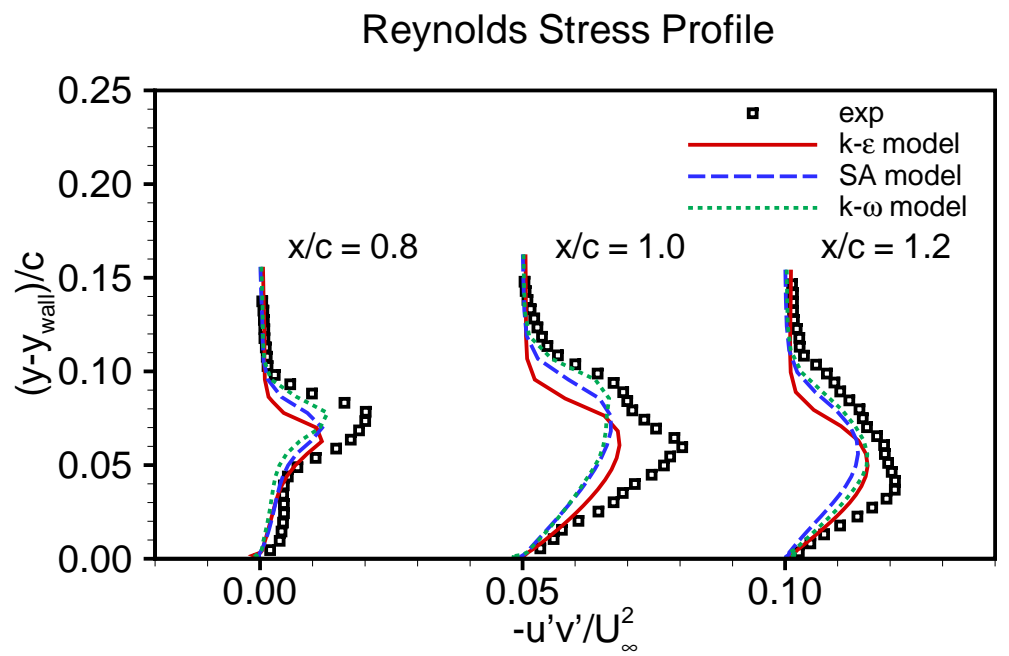

Figure 6. Reynolds shear stress profile predicted by three turbulence models for base flow.

Bodyforce Magnitude Contour



Figure 7. Body force magnitude contour. 


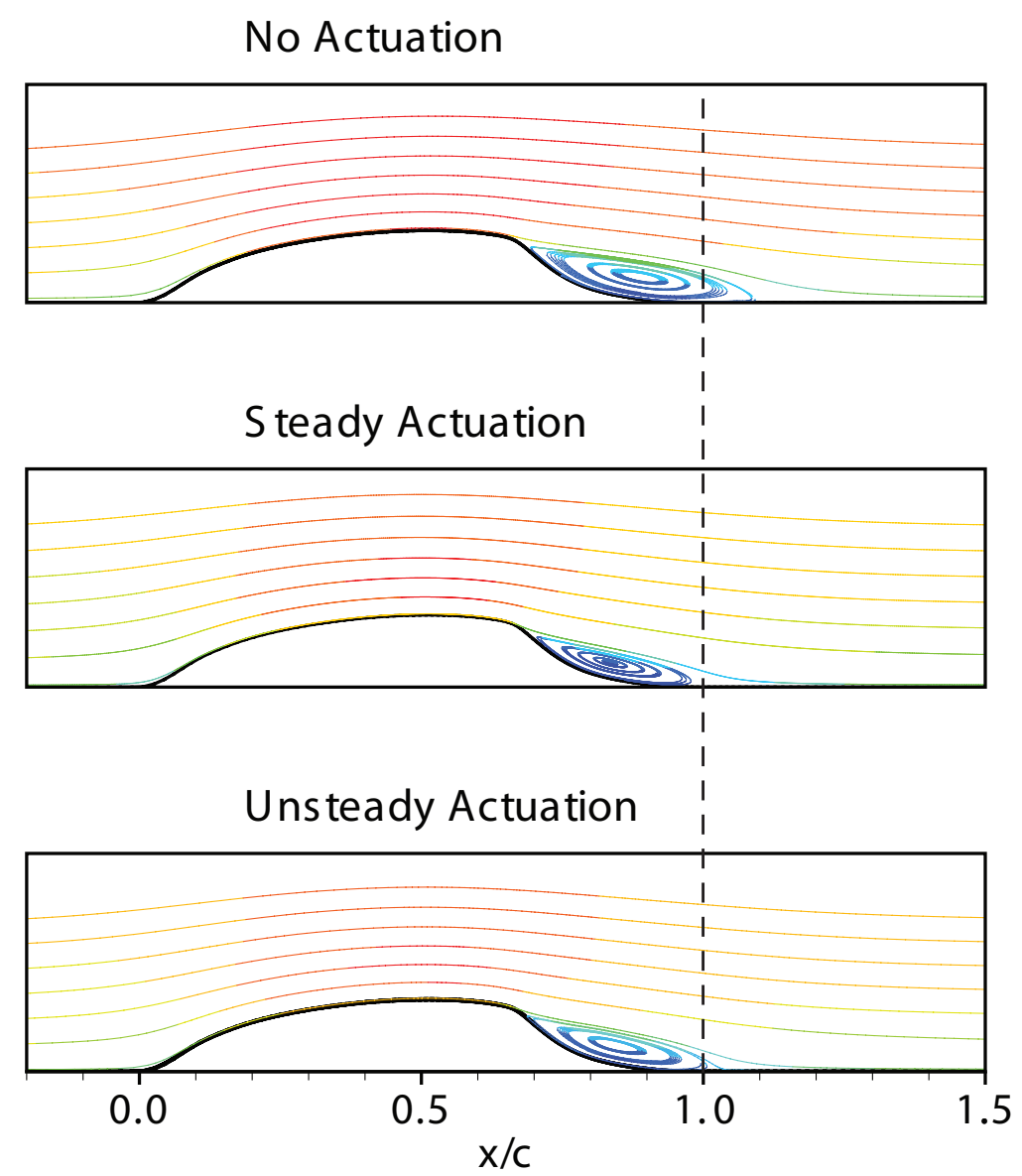

Figure 8. Streamlines for no actuation, steady actuation and unsteady actuation cases, showing the effects of plasma actuator. 




Figure 9. Surface pressure coefficients for uncontrolled and controlled cases predicted by $k-\varepsilon$ model.

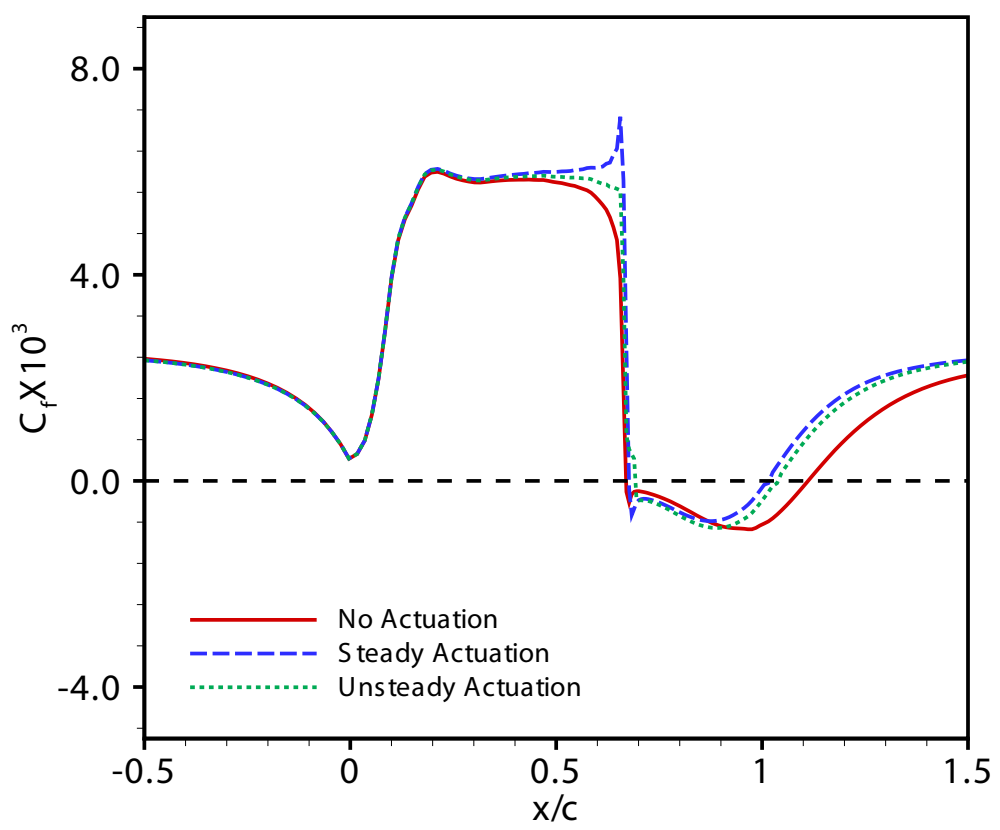

Figure 10. Skin friction coefficients for uncontrolled and controlled cases predicted by $k-\varepsilon$ model. 


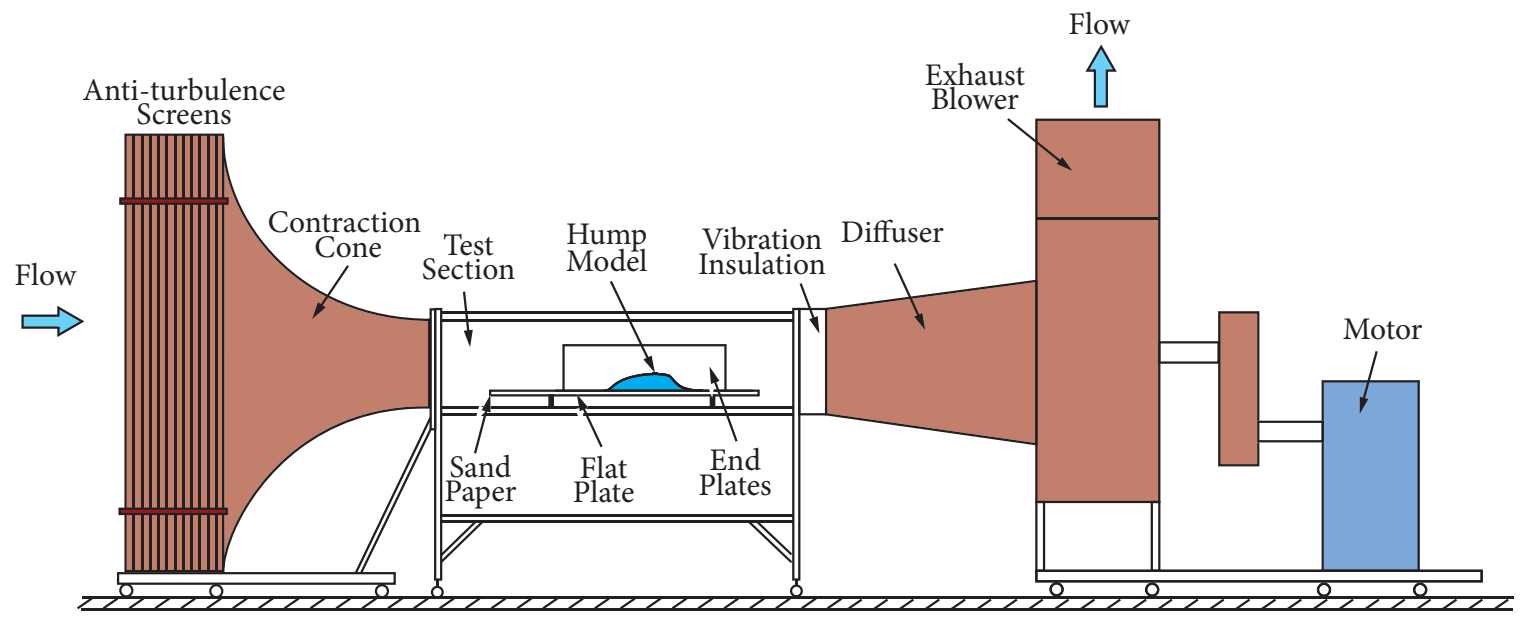

Figure 11. Schematic illustration of subsonic wind tunnel and test section.

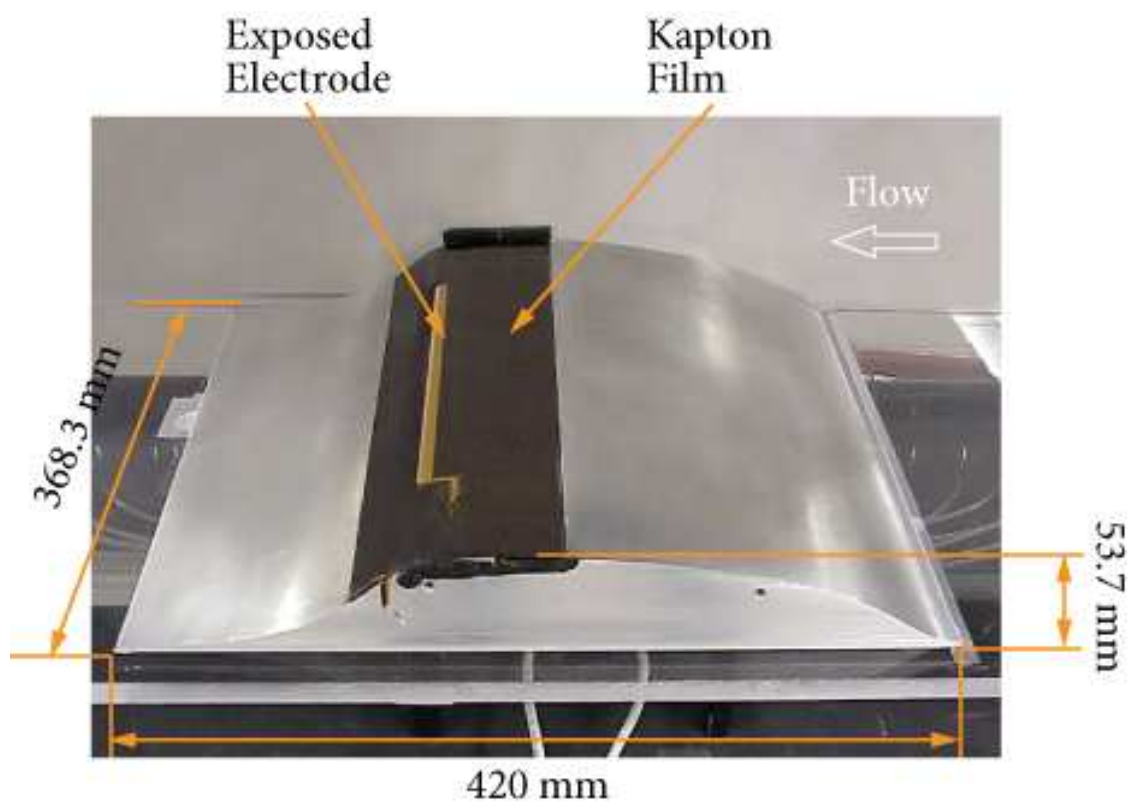

Figure 12. Photograph of wall-mounted hump model and a spanwise plasma actuator on strong convex surface. 




Figure 13. Surface pressure coefficients for base flow and the spanwise plasma actuator at $R e_{c}=288 K$.

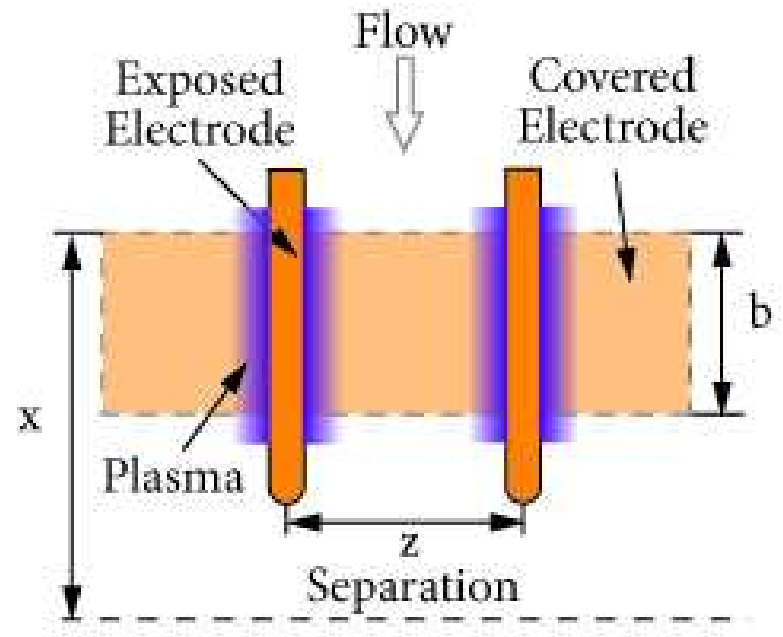

(a)

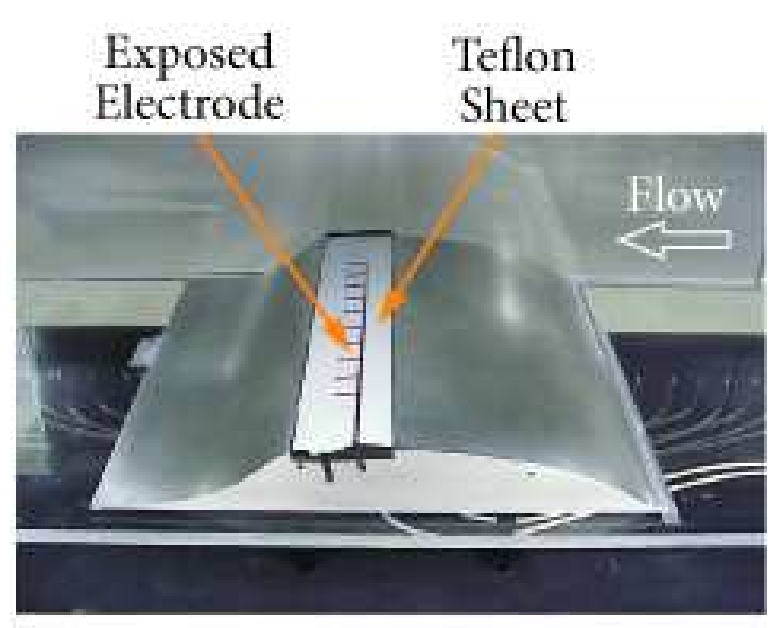

(b)

Figure 14. (a). Schematic illustration of streamwise plasma actuator. (b). Photograph of streamwise plasma actuators. 


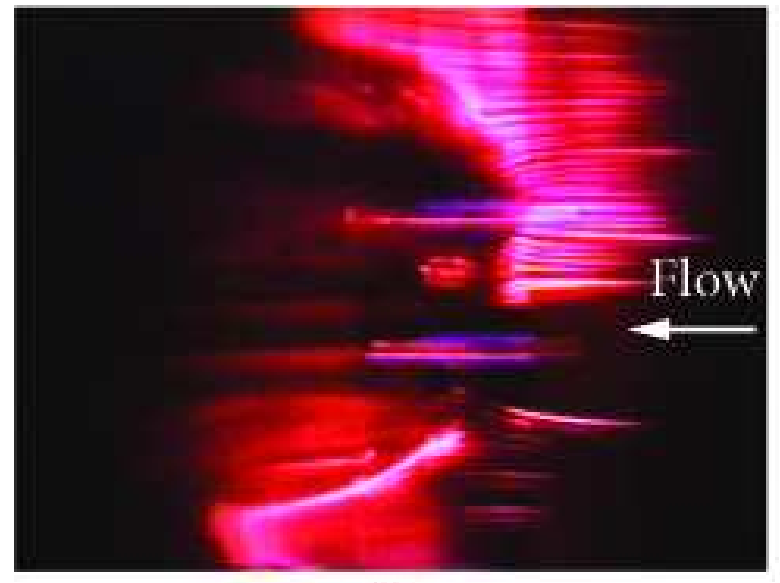

(a)



(b)

Figure 15. Smoke-wire visualization of vortices generated by streamwise plasma actuators. (a) laser sheet and smoke sheet are in the same plane. (b) Laser sheet is higher than the smoke sheet.



Figure 16. Surface pressure coefficient changes as a function of the spacing between exposed electrodes, showing that there is a optimum spacing around $1.5 \mathrm{in}$. 
$\mathrm{V}_{\infty}=10 \mathrm{~m} / \mathrm{s}$, Control with Plasma Actuator

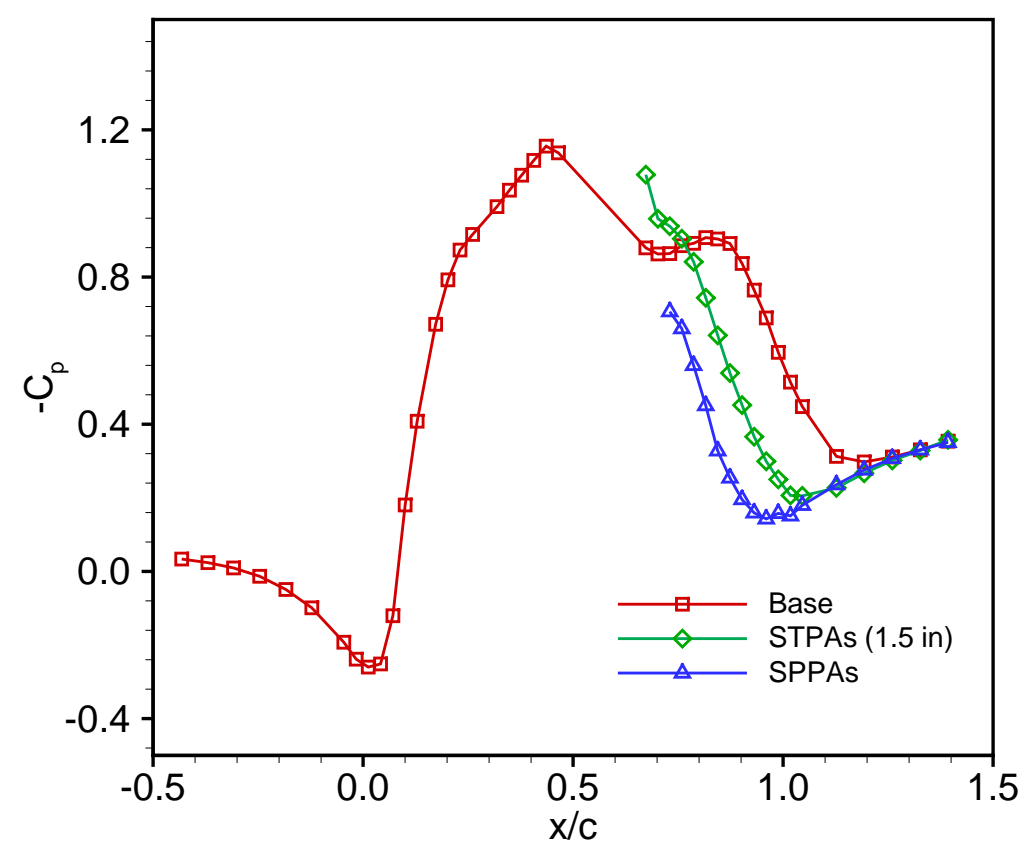

Figure 17. Surface pressure coefficients for base flow, the streamwise plasma actuator and the spanwise plasma actuator at $R e_{c}=288 \mathrm{~K}$. 